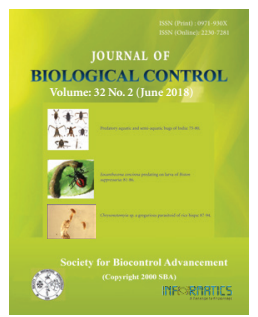

\title{
Evaluation of entomopathogens against blossom midge, Contarinia maculipennis Felt in Jasmine (Jasminum sambac L.)
}

\author{
I. MERLIN KAMALA* and J. S. KENNEDY \\ Department of Agricultural Entomology, Tamil Nadu Agricultural University, Coimbatore -641003, Tamil Nadu, India \\ ${ }^{*}$ Corresponding author E-mail: merlinento@gmail.com
}

\begin{abstract}
The efficacy of microbials against jasmine blossom midge (Contarinia maculipennis Felt) was evaluated under in vitro and field conditions. The results revealed that Metarhizium anisopilae@ $1 \times 10^{8}$ spores $\mathrm{ml}^{-1}$ recorded maximum maggot mortality over untreated control (80.06 per cent) followed by Beauveria bassiana @ $1 \times 10^{8}$ spores $\mathrm{ml}^{-1}$ spores $\mathrm{ml}^{-1}$ recording 67.57 per cent mortality. The Paecilomyces lilacinus @ $1 \times 10^{8}$ spores treated maggots recorded minimum reduction of 50.56 per cent. However, the standard profenophos 50 EC treated maggots recorded 83.78 per cent reduction. Further, field evaluation for confirmation revealed similar trend confirming the efficacy of $M$. anisopilae with minimum infestation of $8.32,7.69$ and 7.31 per cent followed by B. bassiana recording 8.86, 8.93 and 8.50 per cent and L. lecani recording 9.97, 10.26 and 11.77 per cent infestation after first, second and third round of application respectively.
\end{abstract}

KEY WORDS: Blossom midge, Entomopathogens, Formulations, Jasmine

(Article chronicle: Received: 11-11-2017; Revised: 15-04-2018; Accepted: 15-05-2018)

\section{INTRODUCTION}

Jasmine (Jasminum sambac L.) called in Persian as Yasmin i.e., 'Gift of God' is one of the oldest fragrant flowers of India. Flowers and un-opened buds are used for making garlands, bouquets, adorning hairs of women and in religious and ceremonial functions (Thakur et al., 2014). It is used for commercial production of Jasmine concrete, which is the raw material in cosmetic and perfumery industries. As the demand for high grade perfumes has greatly increased in recent times, there is tremendous scope for the development of essential oil from jasmine flowers. In India, jasmine is cultivated in an area of more than 8,000 ha with an annual production of flowers worth Rs. 80-100 million. Tamil Nadu is the leading producer of jasmine in the country with an annual production of 77, 247 tonnes in an area of 9,360 ha (Prakash and Muniandi, 2014). The production of jasmine is affected by various factors; among these insect pests are the most devasting factor. The major pests affecting jasmine are jasmine budworm (Hendecasis duplifascialis (Hampson), galleryworm (Elasmopalpus jasminophagus Hampson.), leaf webworm (Nausinea geometralis Guenee), leaf roller, (Glyphodes unionalis Hubner), blossom midge (Contarinia maculipennis Felt) and two spotted mite (Tetranychus urticae Koch). Of these, budworm and blossom midge gain major economic importance, as they cause excessive damage to the buds. Blossom midge has attained the status of a major pest causing severe economic loss by reducing the marketable quality of the flowers. The midge maggots enter into the buds at the base of the corollas resulting in swelling and shriveling at the base of the buds. The maggots feed inside unopened flower buds, causing deformed, pink discolored buds and blossoms. In case of severe infestations, buds dry prematurely leading to bud drop or blossom drop, thereby the marketable quantity of the flowers are greatly reduced (Fig. 1 and 2).

Jasmine farmers are forced to undertake frequent sprays of pesticides to control the pests. Insecticides were found to be highly effective, rapid in curative action and adoptable to most situations. Frequent application and large-scale use of chemical insecticides for the control of these pests lead to health hazards, outbreaks of secondary pests, environmental pollution, accumulation of pesticide residues and reduction in biodiversity of natural enemies (Balasubramanian and Swamiappan, 1993). Besides, toxic effects of pesticides include emission of unpleasant odours from flowers leading to their rejection by consumers. Further, when exported to foreign countries, the importing countries impound the flowers in the ports, if they detect pesticide residues above MRL. It is pertinent that a change in the insect pest management strategy may form a meaningful solution to avoid the ill-effects caused by the synthetic chemical insecticides especially as environmental contaminants. In recent years, crop protection based on biological control of crop pests with microbial pathogens 


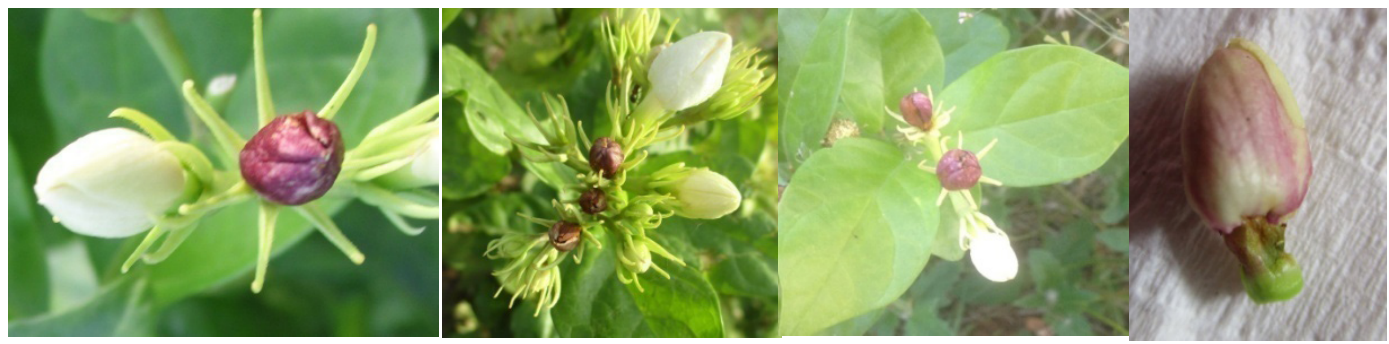

Pink discoloured buds

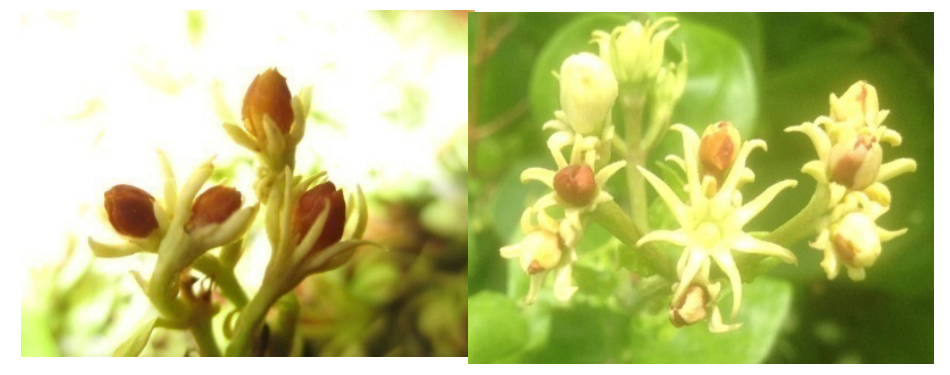

Pre mature dried buds

Fig. 1. Symptoms of damage of blossom midge.

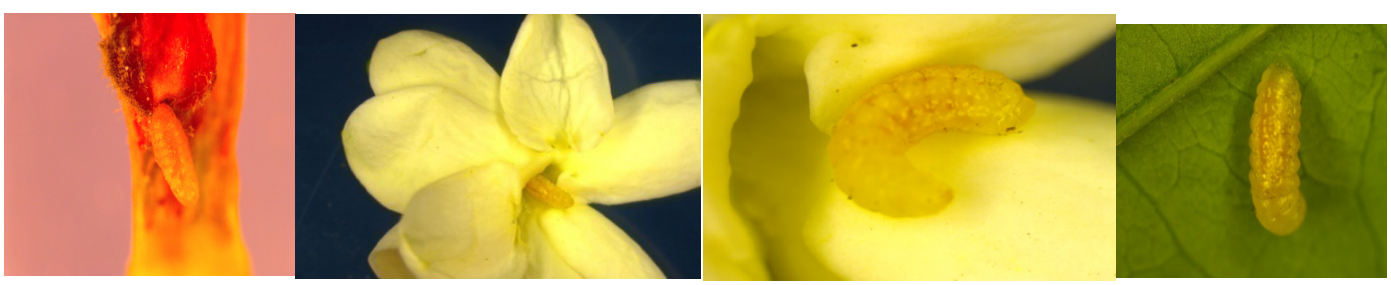

Fig. 2. Midge maggots in jasmine flowers.

like virus, bacteria, fungi and nematodes has been recognized as valuable tools in pest management (Anand et al., 2006, Rao et al., 2006). Research on microbial pathogens of insects is increasing considerably in recent times to find out environmental friendly alternatives to hazardous chemicals. These microbial pesticides occupy around 1.3 per cent of the world's total pesticide market of which, 90 per cent of them are used as insecticides (Menn and Hall, 2001). In light of this understanding, investigations were undertaken to evaluate the microbials especially entomopathogenic fungi which could be economically safer and effective so as to develop environmental friendly pest management strategies against the blossom midge of jasmine.

\section{MATERIALS AND METHODS}

The study on evaluation of microbials on the blossom midge, Contarinia maculipennis was carried out under in vitro and in vivo at the Department of Entomology, Tamil Nadu Agricultural University, Coimbatore under ambient conditions (Temperature, $28 \pm 1{ }^{\circ} \mathrm{C}$ and Relative Humidity
$70 \pm 5 \%$ ) during March-April, 2016 and at farmers holdings at Karamadai village of Coimbatore during May-July, 2016.

\section{Laboratory bioassay}

A laboratory experiment was conducted in a Completely Randomized Design (CRD) with eight treatments replicated thrice. Different microbial formulations used for

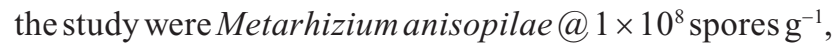

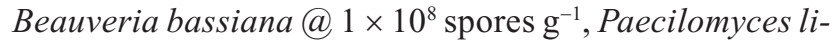
lacinus@ spores g ${ }^{-1}$, Hirsutella thompsonii@ spores ml ${ }^{-1}$, Bacillus thuringiensis@2 $\mathrm{g}^{-1}$ along with a standard insecticide, profenophos50 EC @ $2 \mathrm{ml}^{-1}$ for comparison. In vitro bioassays were conducted to assess the efficacy of microbials on maggots of blossom midge of jasmine by bud dip method. Microbial suspensions were prepared at the desired concentration and placed in a $250 \mathrm{ml}$ conical flask. Field collected infested buds were opened, maggots detached and kept in separate Petri dishes. Jasmine flower buds of equal size were immersed in $50 \mathrm{ml}$ of the microbial suspensions for 30 seconds and the excess fluid was removed by uniform jerking and shade dried. 
Ten buds treated with different concentrations were placed inside Petri dishes lined with moistened filter paper. Ten maggots were introduced to feed on the treated buds into each Petri plate using brushes and observations on maggot mortality were recorded at 24, 48, 72, 96, 120 and 144 hours after treatment.

\section{Field experiment}

A field trial was conducted in farmer's holding at Karamadai, Coimbatore district to evaluate the efficacy of microbials against blossom midge. The experiment was laid out in Randomized Block Design (RBD) with eight treatments including untreated control with plot size $5 \times 4 \mathrm{~cm}^{2}$ replicated thrice. The average temperature of the experimental location was $32^{\circ} \mathrm{C}$ and Relative Humidity 74 per cent. The microbial formulations were bought from International Panaacea Limited. The treatment details are as follows: $\mathrm{T}_{1}-$ Metarhizium anisopliae (Kalichakra), $\mathrm{T}_{2}-$ Beauveria bassiana (Daman), $\mathrm{T}_{3}$ - Paecilomyces lilacinus (Nematofree), $\mathrm{T}_{4}$ - Lecanicillium lecanii (Vertifire), $\mathrm{T}_{5}$ - Hirsutella thompsonii (Almite), $\mathrm{T}_{6}-$ Bacillus thuringiensis var kurstaki (Mahastra), $\mathrm{T}_{7}$ - Profenophos $50 \mathrm{EC}$ (Standard check), T8 - Untreated control. Three rounds of foliar application were given at fortnight interval. The post treatment counts of the midge infestation were recorded on $1,3,7$ and 14 days interval after each spray, besides pretreatment count. The total number of buds in a bush and the number of pink discoloured buds were recorded and the percentage infestation was worked out for statistical scrutiny after suitable transformation.

Table 1. In vitro bio-assay of promising microbials against jasmine blossom midge, Contarinia maculipennis

\begin{tabular}{|c|c|c|c|c|c|c|c|c|c|c|c|}
\hline \multirow[b]{2}{*}{ T.No } & \multirow{2}{*}{ Microbials } & \multirow[b]{2}{*}{ Dosage } & \multirow{2}{*}{$\begin{array}{c}\text { No.of } \\
\text { maggots } \\
\text { released }\end{array}$} & \multicolumn{7}{|c|}{ Maggot count after treatment } & \multirow{2}{*}{$\begin{array}{c}\text { Percent } \\
\text { reduc- } \\
\text { tion over } \\
\text { control }\end{array}$} \\
\hline & & & & $24 \mathrm{hrs}$ & $48 \mathrm{hrs}$ & $72 \mathrm{hrs}$ & $96 \mathrm{hrs}$ & $\begin{array}{l}120 \\
\text { hrs }\end{array}$ & $144 \mathrm{hrs}$ & Mean & \\
\hline $\mathrm{T}_{1}$ & $\begin{array}{l}\text { Metarhizium anisop- } \\
\text { ilae }\end{array}$ & $1 \times 10^{8}$ spores $\mathrm{g}^{-1}$ & 10 & $\begin{array}{c}4.33 \\
(2.08)^{\mathrm{a}}\end{array}$ & $\begin{array}{c}3.66 \\
(1.91)^{b}\end{array}$ & $\begin{array}{c}2.00 \\
(1.41)^{\mathrm{b}}\end{array}$ & $\begin{array}{l}0.66 \\
(0.81)^{\mathrm{a}}\end{array}$ & $\begin{array}{c}0.00 \\
(0.00)^{\mathrm{a}}\end{array}$ & $\begin{array}{c}0.00 \\
(0.00)^{\mathrm{a}}\end{array}$ & $\begin{array}{c}1.77 \\
(1.33)^{\mathrm{ab}}\end{array}$ & 80.06 \\
\hline $\mathrm{T}_{2}$ & Beauveria bassiana & $1 \times 10^{8}$ spores $\mathrm{g}^{-1}$ & 10 & $\begin{array}{c}5.00 \\
(2.23)^{\mathrm{ab}}\end{array}$ & $\begin{array}{c}3.66 \\
(1.91)^{\mathrm{b}}\end{array}$ & $\begin{array}{c}2.00 \\
(1.41)^{\mathrm{b}}\end{array}$ & $\begin{array}{c}1.00 \\
(1.00)^{\mathrm{a}}\end{array}$ & $\begin{array}{c}0.33 \\
(0.57)^{\mathrm{b}}\end{array}$ & $\begin{array}{c}0.00 \\
(0.00)^{\mathrm{b}}\end{array}$ & $\begin{array}{c}1.99 \\
(1.41)^{\mathrm{b}}\end{array}$ & 77.59 \\
\hline $\mathrm{T}_{3}$ & Paecilomyces lilacinus & $1 \times 10^{8}$ spores $\mathrm{g}^{-1}$ & 10 & $\begin{array}{c}6.00 \\
(2.44)^{\mathrm{bcd}}\end{array}$ & $\begin{array}{c}5.33 \\
(2.30)^{\mathrm{cd}}\end{array}$ & $\begin{array}{c}4.66 \\
(2.15)^{\text {de }}\end{array}$ & $\begin{array}{l}3.00 \\
(1.73)^{\mathrm{c}}\end{array}$ & $\begin{array}{c}2.33 \\
(1.52)^{\mathrm{d}}\end{array}$ & $\begin{array}{c}1.00 \\
(1.00)^{\mathrm{d}}\end{array}$ & $\begin{array}{c}3.72 \\
(1.92)^{\mathrm{d}}\end{array}$ & 58.10 \\
\hline $\mathrm{T}_{4}$ & Lecanicillium lecanii & $1 \times 10^{8}$ spores $\mathrm{g}^{-1}$ & 10 & $\begin{array}{c}5.66 \\
(2.37)^{\mathrm{bc}}\end{array}$ & $\begin{array}{c}5.00 \\
(2.23)^{\mathrm{bc}}\end{array}$ & $\begin{array}{c}3.00 \\
(1.73)^{\mathrm{c}}\end{array}$ & $\begin{array}{c}2.33 \\
\left(1.52^{\mathrm{b}}\right.\end{array}$ & $\begin{array}{c}1.00 \\
(1.00)^{\mathrm{c}}\end{array}$ & $\begin{array}{c}0.33 \\
(0.57)^{\mathrm{c}}\end{array}$ & $\begin{array}{c}2.88 \\
(1.69)^{\mathrm{c}}\end{array}$ & 67.57 \\
\hline $\mathrm{T}_{5}$ & Hirsutella thompsonii & $\begin{array}{c}1 \times 10^{8} \text { spores } \\
\mathrm{ml}^{-1} \\
\end{array}$ & 10 & $\begin{array}{c}7.00 \\
(2.64)^{\mathrm{d}} \\
\end{array}$ & $\begin{array}{c}6.33 \\
(2.51)^{d} \\
\end{array}$ & $\begin{array}{c}5.00 \\
(2.23)^{\mathrm{e}} \\
\end{array}$ & $\begin{array}{c}3.66 \\
(1.91)^{\mathrm{c}} \\
\end{array}$ & $\begin{array}{c}3.00 \\
(1.73)^{\mathrm{e}} \\
\end{array}$ & $\begin{array}{c}1.33 \\
(1.15)^{\mathrm{e}} \\
\end{array}$ & $\begin{array}{c}4.39 \\
(2.09)^{\mathrm{d}} \\
\end{array}$ & 50.56 \\
\hline $\mathrm{T}_{6}$ & Bacillus thuringiensis & $0.5 \% \mathrm{~W} . \mathrm{P}$ & 10 & $\begin{array}{c}6.33 \\
(2.51)^{\mathrm{cd}} \\
\end{array}$ & $\begin{array}{c}5.66 \\
(2.38)^{\mathrm{cd}} \\
\end{array}$ & $\begin{array}{c}4.00 \\
(2.00)^{\mathrm{d}}\end{array}$ & $\begin{array}{c}3.33 \\
(1.82)^{\mathrm{c}}\end{array}$ & $\begin{array}{c}2.00 \\
(1.41)^{\mathrm{d}} \\
\end{array}$ & \begin{tabular}{|c|}
0.66 \\
$(0.81)^{\mathrm{d}}$ \\
\end{tabular} & $\begin{array}{c}3.66 \\
(1.91)^{\mathrm{d}} \\
\end{array}$ & 58.78 \\
\hline $\mathrm{T}_{7}$ & Profenophos 50 EC & $2 \mathrm{ml} \mathrm{l}^{-1}$ & 10 & $\begin{array}{c}4.00 \\
(2.00)^{\mathrm{a}}\end{array}$ & $\begin{array}{c}2.66 \\
(1.63)^{\mathrm{a}}\end{array}$ & $\begin{array}{c}1.33 \\
(1.15)^{\mathrm{a}}\end{array}$ & $\begin{array}{c}0.66 \\
(0.81)^{\mathrm{a}}\end{array}$ & $\begin{array}{c}0.00 \\
(0.03)^{\mathrm{a}}\end{array}$ & $\begin{array}{c}0.00 \\
(0.00)^{\mathrm{a}}\end{array}$ & $\begin{array}{c}1.44 \\
(1.20)^{\mathrm{a}}\end{array}$ & 83.78 \\
\hline $\mathrm{T}_{8}$ & Untreated control & - & 10 & $\begin{array}{c}10.00 \\
(3.16)^{\mathrm{e}}\end{array}$ & $\begin{array}{c}10.00 \\
(3.16)^{\mathrm{e}}\end{array}$ & $\begin{array}{c}9.66 \\
(3.10)^{f}\end{array}$ & $\begin{array}{c}9.33 \\
(3.05)^{\mathrm{d}}\end{array}$ & $\begin{array}{c}8.33 \\
(2.88)^{f}\end{array}$ & $\begin{array}{c}6.00 \\
(2.44)^{\mathrm{f}}\end{array}$ & $\begin{array}{c}8.88 \\
(2.97)^{\mathrm{e}}\end{array}$ & \\
\hline \multicolumn{4}{|c|}{ SE CD $(0.05)$} & $\begin{array}{l}0.1146 \\
0.2458\end{array}$ & $\begin{array}{l}0.1097 \\
0.2354\end{array}$ & $\begin{array}{l}0.1005 \\
0.2155\end{array}$ & $\begin{array}{l}0.0907 \\
0.1946\end{array}$ & $\begin{array}{l}0.0823 \\
0.1764\end{array}$ & $\begin{array}{l}0.0646 \\
0.1386\end{array}$ & $\begin{array}{l}0.0948 \\
0.2034\end{array}$ & \\
\hline
\end{tabular}

PROC- Per cent reduction over control

Each value is the mean of three replications.

Figures in parentheses are square root transformed values.

In a column, means followed by common letter (s) is/ are not significantly different by LSD at $\mathrm{P}=0.05 \%$.

Table 2. Field evaluation of promising microbials against blossom midge, Contarinia maculipennis of jasmine - I spray

\begin{tabular}{|c|c|c|c|c|c|c|c|c|c|}
\hline \multirow{2}{*}{ Treatments } & \multirow{2}{*}{ Microbials } & \multirow{2}{*}{ Dosage } & \multirow{2}{*}{$\begin{array}{l}\text { Pre-treated } \\
\text { infestation }\end{array}$} & \multicolumn{5}{|c|}{ I Spray } & \multirow{2}{*}{$\begin{array}{l}\text { Percent re- } \\
\text { duction over } \\
\text { control }\end{array}$} \\
\hline & & & & $1 \mathrm{DAT}$ & 3 DAT & 7 DAT & 15 DAT & Mean & \\
\hline $\mathrm{T}_{1}$ & $\begin{array}{l}\text { Metarrhizium anisop- } \\
\text { ilae }\end{array}$ & $1 \times 10^{8}$ spores $\mathrm{g}^{-1}$ & 31.24 & $\begin{array}{c}9.14 \\
(3.02)^{\mathrm{b}}\end{array}$ & $\begin{array}{c}6.46 \\
(2.54)^{\mathrm{b}}\end{array}$ & $\begin{array}{c}7.79 \\
(2.79)^{\mathrm{a}}\end{array}$ & $\begin{array}{c}9.87 \\
(3.14)^{\mathrm{ab}}\end{array}$ & $\begin{array}{c}8.32 \\
(2.88)^{\mathrm{ab}}\end{array}$ & 73.21 \\
\hline
\end{tabular}




\begin{tabular}{|c|c|c|c|c|c|c|c|c|c|}
\hline $\mathrm{T}_{2}$ & Beauveria bassiana & $1 \times 10^{8}$ spores $\mathrm{g}^{-1}$ & 33.48 & $\begin{array}{c}9.36 \\
(3.06)^{b}\end{array}$ & $\begin{array}{c}6.64 \\
(2.58)^{\mathrm{b}}\end{array}$ & $\begin{array}{c}7.13 \\
(2.67)^{\mathrm{a}}\end{array}$ & $\begin{array}{c}10.08 \\
(3.17)^{\mathrm{b}} \\
\end{array}$ & $\begin{array}{c}8.86 \\
(2.97)^{b}\end{array}$ & 71.52 \\
\hline $\mathrm{T}_{3}$ & Paecilomyces lilacinus & $1 \times 10^{8}$ spores $\mathrm{g}^{-1}$ & 29.47 & $\begin{array}{c}12.23 \\
(3.50)^{\mathrm{cd}}\end{array}$ & $\begin{array}{c}10.35 \\
(3.22)^{\mathrm{c}}\end{array}$ & $\begin{array}{c}13.37 \\
(3.65)^{\mathrm{bc}}\end{array}$ & $\begin{array}{c}15.68 \\
(3.96)^{\mathrm{cd}}\end{array}$ & $\begin{array}{c}12.91 \\
(3.59)^{\text {de }}\end{array}$ & 58.43 \\
\hline $\mathrm{T}_{4}$ & Lecanicillium lecani & $1 \times 10^{8}$ spores $g^{-1}$ & 30.51 & $\begin{array}{c}8.67 \\
(2.94)^{b}\end{array}$ & $\begin{array}{c}9.39 \\
(3.06)^{\mathrm{c}}\end{array}$ & $\begin{array}{c}11.79 \\
(3.43)^{\mathrm{b}}\end{array}$ & $\begin{array}{c}10.04 \\
(3.16)^{b}\end{array}$ & $\begin{array}{c}9.97 \\
(3.15)^{\mathrm{bc}}\end{array}$ & 67.72 \\
\hline $\mathrm{T}_{5}$ & Hirsutella thompsonii & $1 \times 10^{8}$ spores $\mathrm{ml}^{-1}$ & 32.69 & $\begin{array}{c}13.34 \\
(3.65)^{\mathrm{d}}\end{array}$ & $\begin{array}{c}12.43 \\
(3.52)^{\mathrm{d}}\end{array}$ & $\begin{array}{c}15.69 \\
(3.96)^{c}\end{array}$ & $\begin{array}{c}17.87 \\
(4.22)^{\mathrm{d}}\end{array}$ & $\begin{array}{c}14.83 \\
(3.85)^{\mathrm{e}}\end{array}$ & 52.25 \\
\hline $\mathrm{T}_{6}$ & Bacillus thuriengiensis & $0.5 \%$ W.P & 30.48 & $\begin{array}{c}10.34 \\
(3.21)^{\mathrm{bc}}\end{array}$ & $\begin{array}{c}9.75 \\
(3.12)^{\mathrm{c}} \\
\end{array}$ & $\begin{array}{c}11.79 \\
(3.43)^{b}\end{array}$ & $\begin{array}{c}14.47 \\
(3.80)^{\mathrm{c}}\end{array}$ & $\begin{array}{c}11.59 \\
(3.40)^{\mathrm{cd}}\end{array}$ & 62.68 \\
\hline $\mathrm{T}_{7}$ & Profenophos 50 EC & $2 \mathrm{ml} \mathrm{l}^{-1}$ & 26.34 & $\begin{array}{c}6.02 \\
(2.45)^{\mathrm{a}}\end{array}$ & $\begin{array}{c}4.29 \\
(2.07)^{\mathrm{a}}\end{array}$ & $\begin{array}{c}6.14 \\
(2.47)^{\mathrm{a}}\end{array}$ & $\begin{array}{c}7.96 \\
(2.82)^{\mathrm{a}}\end{array}$ & $\begin{array}{c}6.70 \\
(2.58)^{\mathrm{a}}\end{array}$ & 80.36 \\
\hline $\mathrm{T}_{8}$ & Untreated control & & 29.86 & $\begin{array}{c}28.73 \\
(5.35)^{\mathrm{e}} \\
\end{array}$ & $\begin{array}{c}30.17 \\
(5.49)^{\mathrm{e}} \\
\end{array}$ & $\begin{array}{c}31.86 \\
(5.79)^{\mathrm{d}} \\
\end{array}$ & $\begin{array}{c}33.47 \\
(5.78)^{\mathrm{e}} \\
\end{array}$ & $\begin{array}{c}31.06 \\
(5.57)^{\mathrm{e}} \\
\end{array}$ & \\
\hline \multicolumn{3}{|c|}{$\mathrm{SE} C D(0.05) \mathrm{CV} \%$} & NS & $\begin{array}{c}0.140 \\
0 \\
0.3003 \\
5.05\end{array}$ & $\begin{array}{c}0.1379 \\
0.1379 \\
5.28\end{array}$ & $\begin{array}{c}0.149 \\
8 \\
0.3213 \\
5.21\end{array}$ & $\begin{array}{c}0.155 \\
8 \\
0.3343 \\
5.08\end{array}$ & $\begin{array}{c}0.1458 \\
0.3127 \\
5.10\end{array}$ & \\
\hline
\end{tabular}

NS - Non significant: PROC- Percent reduction over control

Each value is the mean of three replications.

Figures in parentheses are arc sine transformed values.

In a column, means followed by common letter ( $s$ ) is / are not significantly different by LSD at $\mathrm{P}=0.05 \%$.

Table 3. Field evaluation of promising microbials against blossom midge, Contarinia maculipennis of jasmine - II spray

\begin{tabular}{|c|c|c|c|c|c|c|c|c|c|}
\hline \multirow{2}{*}{$\begin{array}{c}\text { Treat } \\
\text {-ments }\end{array}$} & \multirow{2}{*}{ Microbials } & \multirow{2}{*}{ Dosage } & \multirow{2}{*}{ PTC } & \multicolumn{5}{|c|}{ II Spray } & \multirow{2}{*}{$\begin{array}{l}\text { Percent re- } \\
\text { duction over } \\
\text { control }\end{array}$} \\
\hline & & & & $1 \mathrm{DAT}$ & 3 DAT & 7 DAT & 15 DAT & Mean & \\
\hline $\mathrm{T}_{1}$ & Metarhizium anisopilae & $1 \times 10^{8}$ spores $\mathrm{g}^{-1}$ & 9.87 & $\begin{array}{c}7.43 \\
(2.72)^{\mathrm{b}}\end{array}$ & $\begin{array}{c}5.78 \\
(2.40)^{\mathrm{b}}\end{array}$ & $\begin{array}{c}6.89 \\
(2.62)^{\mathrm{a}}\end{array}$ & $\begin{array}{c}10.67 \\
(3.26)^{\mathrm{a}}\end{array}$ & $\begin{array}{c}7.69 \\
(2.77)^{\mathrm{ab}}\end{array}$ & 76.39 \\
\hline $\mathrm{T}_{2}$ & Beauveria bassiana & $1 \times 10^{8}$ spores $g^{-1}$ & 14.47 & $\begin{array}{c}8.14 \\
(2.85)^{\mathrm{b}}\end{array}$ & $\begin{array}{c}7.25 \\
(2.69)^{\mathrm{b}}\end{array}$ & $\begin{array}{c}9.04 \\
(3.00)^{\mathrm{bc}}\end{array}$ & $\begin{array}{c}11.27 \\
(3.35)^{\mathrm{a}}\end{array}$ & $\begin{array}{c}8.93 \\
(2.99)^{\mathrm{bc}}\end{array}$ & 72.58 \\
\hline $\mathrm{T}_{3}$ & Paecilomyces lilacinus & $1 \times 10^{8}$ spores $\mathrm{g}^{-1}$ & 17.87 & $\begin{array}{c}12.37 \\
(3.52)^{\mathrm{cd}}\end{array}$ & $\begin{array}{c}11.49 \\
(3.39)^{\mathrm{c}}\end{array}$ & $\begin{array}{c}12.31 \\
(3.51)^{\mathrm{d}}\end{array}$ & $\begin{array}{c}16.86 \\
\left(4.10^{\mathrm{b}}\right.\end{array}$ & $\begin{array}{c}13.26 \\
(3.64)^{d}\end{array}$ & 59.28 \\
\hline $\mathrm{T}_{4}$ & Lecanicillium lecanii & $1 \times 10^{8}$ spores $\mathrm{g}^{-1}$ & 10.04 & $\begin{array}{c}8.47 \\
(2.91)^{\mathrm{b}}\end{array}$ & $\begin{array}{c}7.14 \\
(2.67)^{b}\end{array}$ & $\begin{array}{c}9.68 \\
(3.11)^{c}\end{array}$ & $\begin{array}{c}15.73 \\
(3.96)^{\mathrm{ab}}\end{array}$ & $\begin{array}{c}10.26 \\
(3.20)^{\mathrm{c}}\end{array}$ & 68.50 \\
\hline $\mathrm{T}_{5}$ & Hirsutella thompsonii & $1 \times 10^{8}$ spores $\mathrm{ml}^{-1}$ & 15.68 & $\begin{array}{c}14.65 \\
(3.82)^{\mathrm{d}}\end{array}$ & $\begin{array}{c}12.47 \\
(3.53)^{\mathrm{c}}\end{array}$ & $\begin{array}{c}14.39 \\
(3.79)^{\mathrm{d}}\end{array}$ & $\begin{array}{c}18.71 \\
(4.32)^{b}\end{array}$ & $\begin{array}{c}15.06 \\
(3.88)^{d}\end{array}$ & 53.76 \\
\hline $\mathrm{T}_{6}$ & Bacillus thuringiensis & $0.5 \% W . P$ & 10.08 & $\begin{array}{c}11.26 \\
(3.35)^{\mathrm{c}}\end{array}$ & $\begin{array}{c}10.21 \\
(3.19)^{\mathrm{c}}\end{array}$ & $\begin{array}{c}14.18 \\
(3.76)^{\mathrm{bd}}\end{array}$ & $\begin{array}{c}16.14 \\
(4.01)^{b}\end{array}$ & $\begin{array}{c}12.95 \\
(3.59)^{\mathrm{d}}\end{array}$ & 60.24 \\
\hline $\mathrm{T}_{7}$ & Profenophos 50 EC & $2 \mathrm{ml} \mathrm{l}^{-1}$ & 7.96 & $\begin{array}{c}4.36 \\
(2.09)^{\mathrm{a}} \\
\end{array}$ & $\begin{array}{c}3.78 \\
(1.94)^{\mathrm{a}} \\
\end{array}$ & $\begin{array}{c}5.47 \\
(2.34)^{\mathrm{a}}\end{array}$ & $\begin{array}{c}10.69 \\
(3.27)^{\mathrm{a}}\end{array}$ & $\begin{array}{c}6.08 \\
(2.46)^{\mathrm{a}}\end{array}$ & 81.33 \\
\hline $\mathrm{T}_{8}$ & Untreated control & & 33.47 & $\begin{array}{c}30.51 \\
(5.51)^{\mathrm{e}} \\
\end{array}$ & $\begin{array}{c}31.49 \\
(5 . .60)^{d}\end{array}$ & $\begin{array}{c}33.57 \\
(5.78)^{\mathrm{e}} \\
\end{array}$ & $\begin{array}{c}34.69 \\
(5.88)^{\mathrm{c}} \\
\end{array}$ & $\begin{array}{c}32.57 \\
(5.70)^{\mathrm{e}}\end{array}$ & \\
\hline & $\mathrm{SE} \mathrm{CD}(0.05)$ & & NS & $\begin{array}{l}0.1725 \\
0.3699\end{array}$ & $\begin{array}{l}0.1195 \\
0.2493\end{array}$ & $\begin{array}{l}0.1830 \\
0.3926\end{array}$ & $\begin{array}{l}0.1985 \\
0.4258\end{array}$ & $\begin{array}{l}0.1811 \\
0.3884\end{array}$ & \\
\hline
\end{tabular}

NS - Non significant: PROC- Per cent reduction over control

Each value is the mean of three replications.

Figures in parentheses are arc sine transformed values.

In a column, means followed by common letter (s) is / are not significantly different by LSD at P $=0.05 \%$. 


\section{Statistical analysis}

For laboratory bio-assays, the data on maggot count were subjected to $\sqrt{\mathrm{x}}$ transformation and the treatment mean values were compared using Latin Square Distribution (LSD). The corrected per cent mortality was worked out by using Abbott's correction. The method suggested by Gomez and Gomez, (1984) was adopted for statistical analysis of the data obtained from the field experiment. Prior to analysis, the data were subjected to $\sqrt{\mathrm{x}}$ transformation and the mean values of treatments were then compared using Latin Square Distribution (LSD).

\section{RESULTS AND DISCUSSION}

Microbial (bio) insecticides, products based on living micro-organisms which are pathogenic to insects, are gaining importance in entomological research of pest management lately. With the pros and cons of synthetic insecticides, Integrated Pest Management (IPM) comes as a direct tool that includes array of alternatives like exploration of botanicals and microbial formulations. The microbials evaluated against Contarinia maculipennis under laboratory condition showed a varied response on their insecticidal activity (Table 1). Among the six microbial formulations tested, Metarhizium anisopliae showed maximum mean maggot mortality over untreated control of 80.06 per cent followed by Beauveria bassiana recording 77.59 per cent and Lecanicillium lecanii recording 67.57 per cent of maggot mortality. Field experiment conducted to evaluate the efficacy of certain promising microbial pesticides against blossom midge revealed the similar trend as laboratory evaluations. The pretreatment infestation of pink buds ranged from 26.34 to 33.48 per cent. After the first spray (Table 2) in M. anisopliae imposed plots, the infestation reduced from a pretreatment count of 31.24 per cent to 9.14 per cent on the first day, it was further reduced to 6.46 per cent on the third day, recording a maximum of 73.21 per cent mean reduced infestation over untreated control $B$. bassiana treated plots recorded 71.52 percent reduced infestation and L. lecanii treated plot s recording 67.72 per cent falls next in the order. The other microbials tested viz., Bacillus thuringiensis var kurstaki (Bt) and Paecilomyces lilacinus were on par in their efficacy recording 58.78 and 58.10 per cent mortality respectively. Hirsutella thompsonii showed least efficacy ( 50.56 per cent) in managing midge maggots and the standard check, profenophos recorded maximum of 84.45 per cent maggot mortality. The second spray (Table 3 ) was given 15 days after the first spray and the pre-treated infestation of pink buds ranged from 7.96 to 33.47 per cent. The M. anisopliae and B. bassiana treated plots were on par in their efficacy recording 76.39, and 72.58 percent reduction over untreated control. The $L$. lecanii treated plots recorded 68.50 per cent infestation, $B t$ and P. lilacinus treated plots recorded 60.24 and 59.28 per cent infestation and were statistically on par and came next in the order of efficacy. H. thompsonii recorded only 53.76 per cent reduction in infestation falling last in the order of efficacy. The standard synthetic chemical profenophos maintained its superiority with 81.33 per cent reduction of infestation over control. The third spray (Table 4) was given 15 days after the second spray and a pre-treated infestation of 10.67 to 34.69 per cent was recorded. Similar trend of efficacy was exposed with $M$. anisopliae treated plots recording maximum per cent reduction over control of 79.52 per cent infestation followed by $B$. bassiana imposed plots with 75.83 per cent reduction over control and was statistically on par. The order of efficacy follows as L. lecanii imposed plots (66.54), P. lilacinus (59.89), Bt (55.91) and H. thomponii (53.97). However, the synthetic chemical profenophos imposed plots recorded the highest per cent reduction over control of 81.75 percent.

The three microbial sprays given in jasmine field revealed the efficacy of $M$. anisopliae in managing jasmine blossom midge followed by B. bassiana and L. lecanii. The results are in accordance with Bruck (2006) who stated that Metarhizium aniopliae @ $1 \times 10^{6} \mathrm{cfu} / \mathrm{g}$ was found to be effective in controlling rose midges, Dasineura rhodophaga. Nicholas and Mc Corkell (2014) proved that the incorporation of $M$. anisopliae into freshly excreted cattle dung at rates of between 0.25 and $1 \mathrm{~g}$ conidia $/ \mathrm{kg}$ reduced the emergence of adult midges by up to $98.5 \%$ compared to untreated dung indicating that $M$. anisopliae has the potential to control C. brevitarsis larvae in cattle dung. Furthermore, laboratory bioassays according to Toledo et al. (2006) showed that several strains of M. anisopliae (Ma3, Ma5 y, Ma wild) and B. bassiana from the ECOSUR strain collection were highly virulent to adult fruitflies of Anastrepha ludens and Ceratistis capitata. Moreover, Narladkar (2015) explored the potency of the fungal spores of $M$. anisopliae and $B$. bassiana against larval and adult Culicoides midges, a pest of livestock. The least mean per cent reduction in infestation over control was recorded in H. thompsonii 52.25, 53.76 and 53.97 per cent reduction followed by $P$. lilacinus treated plots with $58.43,59.28$ and 59.89 per cent reduction. The reason for the less effectiveness of $H$. thompsonii must be its acaricidal property, which is effective againt mites and the nematicidal properties of $P$. lilacinus is effective in controlling nematodes rather than insects. 
Table 4. Field evaluation of promising microbials against blossom midge, Contarinia maculipennis of jasmine - III

\begin{tabular}{|c|c|c|c|c|c|c|c|c|c|}
\hline \multirow{2}{*}{$\begin{array}{l}\text { Treat- } \\
\text { ments }\end{array}$} & \multirow{2}{*}{ Microbials } & \multirow{2}{*}{ Dosage } & \multirow{2}{*}{ PTC } & \multicolumn{5}{|c|}{ III Spray } & \multirow{2}{*}{$\begin{array}{l}\text { Percent } \\
\text { reduction over } \\
\text { control }\end{array}$} \\
\hline & & & & $1 \mathrm{DAT}$ & 3 DAT & 7 DAT & 15 DAT & Mean & \\
\hline $\mathrm{T}_{1}$ & Metarrhizium anisopilae & $1 \times 10^{8}$ spores $\mathrm{g}^{-1}$ & 10.67 & $\begin{array}{c}6.46 \\
(2.54)^{\mathrm{a}}\end{array}$ & $\begin{array}{c}5.17 \\
(2.27)^{\mathrm{ab}}\end{array}$ & $\begin{array}{c}7.21 \\
(2.68)^{\mathrm{ab}}\end{array}$ & $\begin{array}{c}10.39 \\
(3.22)^{\mathrm{a}}\end{array}$ & $\begin{array}{c}7.31 \\
(2.70) \mathrm{a}\end{array}$ & 79.22 \\
\hline $\mathrm{T}_{2}$ & Beauveria bassiana & $1 \times 10^{8}$ spores $\mathrm{g}^{-1}$ & 16.14 & $\begin{array}{c}7.56 \\
(2.75)^{\mathrm{b}}\end{array}$ & $\begin{array}{c}6.21 \\
(2.49)^{\mathrm{b}}\end{array}$ & $\begin{array}{c}8.74 \\
(2.95)^{\mathrm{b}}\end{array}$ & $\begin{array}{c}11.47 \\
(3.38)^{\mathrm{ab}}\end{array}$ & $\begin{array}{c}8.50 \\
(2.91)^{\mathrm{a}}\end{array}$ & 75.83 \\
\hline $\mathrm{T}_{3}$ & Paecilomyces lilacinus & $1 \times 10^{8}$ spores $\mathrm{g}^{-1}$ & 18.71 & $\begin{array}{c}12.47 \\
(3.53)^{\mathrm{cd}}\end{array}$ & $\begin{array}{c}11.13 \\
(3.33)^{\mathrm{cd}}\end{array}$ & $\begin{array}{c}14.15 \\
(3.76)^{\mathrm{cd}}\end{array}$ & $\begin{array}{c}18.67 \\
(4.32)^{\mathrm{c}}\end{array}$ & $\begin{array}{c}14.11 \\
(3.75)^{\mathrm{bc}}\end{array}$ & 59.89 \\
\hline $\mathrm{T}_{4}$ & Lecanicillium lecani & $1 \times 10^{8}$ spores $\mathrm{g}^{-1}$ & 15.73 & $\begin{array}{c}10.24 \\
(3.19)^{\mathrm{c}}\end{array}$ & $\begin{array}{c}10.01 \\
(3.16)^{\mathrm{c}}\end{array}$ & $\begin{array}{c}12.47 \\
(3.52)^{\mathrm{c}}\end{array}$ & $\begin{array}{c}14.34 \\
(3.78)^{\mathrm{b}}\end{array}$ & $\begin{array}{c}11.77 \\
(3.42)^{\mathrm{ab}}\end{array}$ & 66.54 \\
\hline $\mathrm{T}_{5}$ & Hirsutella thompsonii & $1 \times 10^{8}$ spores $\mathrm{ml}^{-1}$ & 16.86 & $\begin{array}{c}14.16 \\
(3.76)^{d}\end{array}$ & $\begin{array}{c}13.69 \\
(3.70)^{\mathrm{d}}\end{array}$ & $\begin{array}{c}15.82 \\
(3.97)^{\mathrm{d}}\end{array}$ & $\begin{array}{c}21.07 \\
(4.59)^{\mathrm{c}}\end{array}$ & $\begin{array}{c}16.19 \\
(4.02)^{\mathrm{c}}\end{array}$ & 53.97 \\
\hline $\mathrm{T}_{6}$ & Bacillus thuriengiensis & $0.5 \%$ W.P & 11.27 & $\begin{array}{c}13.47 \\
(3.66)^{\text {bd }}\end{array}$ & $\begin{array}{c}12.49 \\
(3.53)^{\mathrm{cd}}\end{array}$ & $\begin{array}{c}15.78 \\
(3.96)^{d}\end{array}$ & $\begin{array}{c}20.29 \\
(4.50)^{\mathrm{c}}\end{array}$ & $\begin{array}{c}15.51 \\
(3.93)^{\mathrm{c}}\end{array}$ & 55.91 \\
\hline $\mathrm{T}_{7}$ & Profenophos 50 EC & $2 \mathrm{ml} / \mathrm{lit}$ & 10.69 & $\begin{array}{c}5.14 \\
(2.26)^{\mathrm{a}} \\
\end{array}$ & $\begin{array}{c}4.26 \\
(2.06)^{\mathrm{a}} \\
\end{array}$ & $\begin{array}{c}6.48 \\
(2.54)^{\mathrm{a}} \\
\end{array}$ & $\begin{array}{c}9.78 \\
(3.12)^{\mathrm{a}} \\
\end{array}$ & $\begin{array}{c}6.42 \\
(2.53)^{\mathrm{a}} \\
\end{array}$ & 81.75 \\
\hline $\mathrm{T}_{8}$ & Untreated control & & 34.69 & $\begin{array}{c}34.14 \\
(5.83)^{\mathrm{e}}\end{array}$ & $\begin{array}{c}33.71 \\
(5.79)^{\mathrm{e}}\end{array}$ & $\begin{array}{c}35.49 \\
(5.95)^{\mathrm{e}}\end{array}$ & $\begin{array}{c}37.38 \\
(6.10)^{\mathrm{d}}\end{array}$ & $\begin{array}{c}35.18 \\
(5.92)^{d}\end{array}$ & \\
\hline & $\mathrm{SE} C D(0.05)$ & & & $\begin{array}{l}0.1826 \\
0.3917\end{array}$ & $\begin{array}{l}0.1788 \\
0.3835\end{array}$ & $\begin{array}{l}0.1916 \\
0.4109\end{array}$ & $\begin{array}{l}0.2070 \\
0.4440\end{array}$ & $\begin{array}{l}0.1903 \\
0.4082\end{array}$ & \\
\hline
\end{tabular}

NS - Non significant: PROC- Percent reduction over control

Each value is the mean of three replications.

Figures in parentheses are arc sine transformed values.

In a column, means followed by common letter (s) is / are not significantly different by LSD at

The present study revealed that the microbial agent, Metarhizium anisopliae in three rounds of spray at 14 days interval was quite effective in bringing down the damage by Contarinia maculipennis and increasing the flower yield of jasmine and can be considered as potent alternative to synthetic insecticides. Beauveria bassiana and Lecanicillium lecanii also proved their efficacy in the next level in managing blossom midge of jasmine. Hence use of $M$. anisopliae will be useful in the attempt to combat the ill effects of synthetic insecticides.

\section{ACKNOWLEDGEMENT}

The financial assistance provided by UGC, as fellowship to the senior author to pursue Ph.D. in Agricultural Entomology at Tamil Nadu Agricultural University, Coimbatore is gratefully acknowledged.

\section{REFERENCES}

Anand R, Prasad B, Tiwary BN. 2009. Relative susceptibility of Helicoverpa armigera pupae to selected entomopathogenic fungi. Biol Control 54: 85-92.
Bruck T. 2006. Metarhizium anisopliae for management of rose midge. Project submitted to Oregon State University.

Balasubramanian V, Swamiappan, M. 1993. Screening of insecticides used in cotton ecosystem for their effect on the green lacewing, Chrysoperla carnea Stephens (Neuroptera: Chrysopidae) In: National Symposium on Advances in Biological Control of Insect Pests, 2-4, Oct, 1993. Uttar Pradesh Zoological Society. pp. 30 .

Menn JJ, Hall FR. 2001. Biopesticides- Present status and future prospects. In: Menn JJ and Hall FR (Eds.). Methods in Biotechnology, Vol. 5: Biopesticides Use and Delivery, Totowa, NJ: Humana Press, pp. 1-9.

Narladkar BW, Shivpuje PR, Harke PC. 2015. Fungal biological control agents for integrated management of Culicoides spp. (Diptera: Ceratopogonidae) of livestock. Vet World. 8(2): 156-163. https:// doi.org/10.14202/vetworld.2015.156-163 PMid:27047065 PMCid:PMC4774696 
Nicholas AH, McCorkell B. 2014. Evaluation of Metarhizium anisopliae for the control of Culicoides brevitarsis Kieffer (Diptera: Ceratopogonidae), the principal vector of bluetongue virus in Australia. $J$. Vector Ecol. 39(1): 213-8. https://doi.org/10.1111/ j.1948-7134.2014.12089.x PMid:24820575

Prakash K, Muniyandi B. 2014. Application of ARIMA model for forecasting production of jasmine flower in Madurai District of Tamil Nadu, India. Am Int J Res Humanit Arts Soc Sci. 14: 279-285.

Rao C, Devi KU, Khan PAA. 2006. Effect of combination treatment with entomopathogenic fungi, Beauveria bassiana and Nomuraea rileyi (Hypocreales) on Helicoverpa armigera (Lepidoptera: Noctuidae). Biocontrol Sci Tech. 16: 221-232. https://doi. org/10.1080/09583150500335632
Thakur A, Naqvi SMA, Aske DK, Sainkhediya. 2014. Study of some ethno medicinal plants used by Tribals of Alirajpur, Madhya Pradesh, India. Res J Agric Forestry Sci. 2(4): 9-12.

Toledo J, Pablo L, Flores S, Campos SE, Villasenor A, Montoya, P. 2006. Use of Beauveria bassiana and Metarhizium anisopliae for fruit fly control: A novel approach. Fruit Flies of Economic Importance: From Basic to Applied Knowledge. Proceedings of the 7th International Symposium on Fruit Flies of Economic Importance 10-15 September 2006, Salvador, Brazil. pp. $127-132$. 